\title{
DOSE ECONÔMICA DE CALCÁRIO NA PRODUTIVIDADE DE CARAMBOLEIRAS ${ }^{1}$
}

\author{
WILLIAM NATALE 2 , DANILO EDUARDO ROZANE ${ }^{3}$, RENATO DE MELLO PRADO ${ }^{2}$, \\ LILIANE MARIA ROMUALDO ${ }^{3}$, HENRIQUE ANTUNES DE SOUZA ${ }^{3}$, AMANDA HERNANDES
}

RESUMO - Com o objetivo de estudar a relação entre doses de calcário e produção de carambolas, um experimento de campo foi conduzido em um Latossolo Vermelho distrófico, no município de Bebedouro(SP), no período de 1999 a 2006. O delineamento adotado foi o em blocos casualizados, com cinco tratamentos e quatro repetições. O calcário foi aplicado uma única vez em 1999, nas doses de: 0; 1,85; 3,71; 5,56 e 7,41 t $\mathrm{ha}^{-1}$. A produção das caramboleiras aumentou em resposta à aplicação do corretivo de acidez. As produções acumuladas de frutos, nos anos sucessivos de 2003, 2004, 2005 e 2006, estiveram associadas às doses mais econômicas de calcário de 4,5; 4,8; 5,3 e 5,3 $\mathrm{t} \mathrm{ha}^{-1}$, respectivamente.

Termos para indexação: Averrhoa carambola, acidez do solo, calagem, dose econômica.

\section{ECONOMICAL DOSE OF LIMING IN YIELD OF STAR FRUIT}

\begin{abstract}
With the objective to study the relationship between limestone doses and star fruit production, a field experiment was conducted on Oxisol soil in Bebedouro, São Paulo state, Brazil, from 1999 to 2006. The experimental design was a randomized blocks with four replications. The limestone was used only once in 1999 , at doses from $0,1.85 ; 3.71 ; 5.56$ and 7.41 ton $\mathrm{ha}^{-1}$. The guava production increased in response to the acidity corrective application. The accumulated production of fruits in successive years 2003, 2004, 2005 and 2006 was associated with economical doses of limestone of 4.5; 4.8; 5.3 and 5.3 ton ha ${ }^{-1}$ respectively.
\end{abstract}

Index terms: Averrhoa carambola; soil acidity; limestone; economic dose.

\section{INTRODUÇÃO}

O crescimento mundial da população e de sua renda nas últimas décadas tem provocado aumento no consumo de insumos a fim de atender à demanda de alimentos. Isso afeta diretamente a agricultura em geral e, em particular, a produção de frutas.

O Brasil possui grandes áreas cultivadas com frutíferas (2,6 milhões de hectares), sendo um dos maiores produtores mundiais $(43,6$ milhões de toneladas/ano) de frutas (FAO, 2011). Entretanto, as produtividades em geral são baixas e dependem da adequada nutrição mineral dos pomares para atingir níveis satisfatórios de colheitas. Contudo, um diagnóstico adequado das necessidades das culturas permite aplicações racionais de corretivo nos pomares e maior eficiência no aproveitamento dos fertilizantes.

A caramboleira é uma frutífera exótica com grande potencial para exploração nas condições brasileiras. Os frutos, além de atenderem às principais características apreciadas pelo consumidor, tais como a cor, o sabor, o aroma e as propriedades nutricionais, destacam-se pela sua beleza exótica, o que os torna bastante atrativos (DONADIO et al., 2001).

A caramboleira, assim como as árvores frutíferas e todas as perenes, permanece longos períodos explorando praticamente o mesmo volume de solo, razão pela qual o ambiente radicular, em especial com relação à acidez, característica comum nos solos brasileiros, merece a máxima atenção. Esse grupo de plantas tem imensa capacidade de armazenamento de elementos essenciais em sua vegetação, além de possuir estabilidade nutricional na fase adulta. Apesar disso, as enormes quantidades de nutrientes exportados pelas colheitas, a cada safra, podem diminuir as concentrações no solo. Aliado a isso, a acidificação do solo é um processo natural (RAIJ, 1991) que, somado à ação antrópica para atender à demanda das caramboleiras, o qual o solo não é capaz de suprir, a exemplo, da adição de fertilizantes nitrogenados, pode acelerar este processo (LEAL et al., 2007).

Assim, a correção da acidez do solo do pomar, por meio da prática da calagem, permite intervenções racionais do ponto de vista agronômico e econô-

\footnotetext{
${ }^{1}$ (Trabalho 034-11). Recebido em: 05-01-2011. Aceito para publicação em: 05-04-2011.

2Professor Adjunto, Departamento de Solos e Adubos, Universidade Estadual Paulista "Julio de Mesquita Filho", Faculdade de Ciências Agrárias e Veterinárias (FCAV/Unesp). Via de Acesso Professor Paulo Donato Castellane, s/n. 14884-900, Jaboticabal-SP. E-mails: natale@fcav.unesp.br; rmprado@fcav.unesp.br

${ }^{3}$ Professor Doutor, Universidade Estadual Paulista "Julio de Mesquita Filho", Campus Experimental de Registro. Rua Nelson Brihi Badur, n 430. CEP 11900-000, Registro - SP. E-mail: danilorozane@registro.unesp.br Pós-Graduanda - Faculdade de Zootecnia e Engenharia de Alimentos, USP, Campus Pirassununga. E-mail: lilianeromualdo@yahoo.com.br
} 
mico, melhorando o desenvolvimento do sistema radicular (PRADO; NATALE, 2004a), e o acúmulo de nutrientes nas folhas de caramboleiras (PRADO; NATALE, 2004b), devido às melhorias nas condições da fertilidade do solo que, consequentemente, promovem incremento de produção (NATALE et al., 2008). Desse modo, deve ser melhor ponderada a relação beneficio/custo, seja pelo uso equilibrado do corretivo ou pela economia de fertilizantes, seja pelo incremento da produtividade.

Segundo Raij (1991), a lei dos incrementos decrescentes serve de base para a definição da dose mais econômica de um insumo. Quando o valor do aumento em produção é igual ao custo do corretivo, atinge-se um nível de aplicação acima do qual a calagem não se traduz em retorno econômico e que corresponde à chamada dose econômica.

Há poucos estudos sobre calagem em cultivo de frutíferas, especialmente considerando longos períodos de experimentação. Além disso, são inexistentes pesquisas avaliando a dose mais econômica de corretivo a ser aplicada na implantação dos pomares, o que é de fundamental importância, a fim de evitar super ou subcalagem, aperfeiçoar a alocação desse insumo na produção de frutos e, consequentemente, garantir retorno econômico.

Em função do exposto, e como parte de um estudo mais amplo sobre a cultura da carambola, procurou-se, por meio da realização de um experimento de campo, conduzido no período de 1999 a 2006, avaliar os efeitos de diferentes doses de calcário sobre a produção de frutos, calculando-se a dose economicamente viável de corretivo para a implantação de um pomar de caramboleiras.

\section{MATERIAL E MÉTODOS}

O experimento foi conduzido em área experimental pertencente à Estação Experimental de Citricultura de Bebedouro, na região de Bebedouro (SP), com coordenadas geográficas $20^{\circ} 53^{\prime} \mathrm{S}$ de latitude e $48^{\circ} 28^{\prime} \mathrm{W}$ de longitude, a uma altitude de 601 metros. O clima local é do tipo Cwa subtropical, segundo a classificação de Köeppen, com inverno curto, moderado e seco, e verão quente e chuvoso, caracterizando duas estações distintas.

O solo é um Latossolo Vermelho distrófico típico (EMBRAPA, 1999).Os atributos químicos do solo, antes da implantação do experimento, estão apresentados na Tabela 1 .

O delineamento experimental utilizado foi em blocos casualizados, com cinco tratamentos e quatro repetições, constituídos de doses crescentes de calcário $\left(\mathrm{CaO}=456\right.$ e $\left.\mathrm{MgO}=102 \mathrm{~g} \mathrm{~kg}^{-1}\right)$, com PRNT
$=100 \%$. As doses foram calculadas considerando-se os resultados médios da análise química do solo das camadas de $0-20$ e $20-40 \mathrm{~cm}$ (Tabela 1) e, também, para a incorporação do corretivo na camada de 0-30 $\mathrm{cm}$. Tomou-se como referência a dose calculada para atingir a saturação por bases $(\mathrm{V})$ igual a $70 \%(3,71 \mathrm{t}$ $\mathrm{ha}^{-1}$ ), sendo considerada média, no limite superior das classes de interpretação relacionadas com a acidez da camada arável do solo (RAIJ et al., 1997), uma vez que não havia recomendação para a caramboleira e, a partir desta, como segue: $\mathrm{D}_{0}=$ zero; $\mathrm{D}_{1}=$ metade da dose recomendada; $\mathrm{D}_{2}=$ a dose total recomendada; $\mathrm{D}_{3}=1,5 \mathrm{vez}$ a dose recomendada; $\mathrm{D}_{4}=2$ vezes a dose necessária para elevar $\mathrm{V}$ a $70 \%$, correspondendo a: $0 ; 1,85 ; 3,71 ; 5,56$ e $7,41 \mathrm{t} \mathrm{ha}^{-1}$, respectivamente.

A aplicação do calcário foi realizada de forma manual na superfície do solo, em julho/agosto de 1999, metade antes da incorporação com arado de aivecas, e a outra metade aplicado e incorporado com grade aradora, abrangendo a camada de 0-30 $\mathrm{cm}$. Após quatro meses, foi implantado o pomar, no espaçamento de $7 \mathrm{~m}$ entre linhas e 4,2 m entre plantas, utilizando-se de caramboleiras (Averrhoa carambola L.), enxertadas (cv. Malásia). As parcelas foram compostas por cinco plantas, sendo consideradas as três centrais como úteis para as avaliações. A adubação básica na cova de plantio da frutífera constou da mistura de $180 \mathrm{~g}$ de $\mathrm{P}_{2} \mathrm{O}_{5}$ na forma de superfosfato simples, $20 \mathrm{~L}$ de composto orgânico, além de $1 \mathrm{~g}$ de $\mathrm{B}$ e $2 \mathrm{~g}$ de $\mathrm{Zn}$, respectivamente, na forma de ácido bórico e de sulfato de zinco.

A adubação durante todo o período experimental foi adaptada das indicações de Natale et al. (1996), considerando-se os atributos químicos do solo, a idade do pomar e a produção esperada de frutos.

O sistema de irrigação foi instalado seis meses após o transplantio das mudas, colocando-se um microaspersor por planta, com vazão de $26 \mathrm{~L} \mathrm{~h}^{-1}$, acionando-se o sistema quando eram consumidos cerca de $30 \%$ da disponibilidade de água no solo avaliado por tensiometria (BERNARDO, 2002) .

Avaliou-se a produção das três plantas úteis de cada parcela, durante cinco anos agrícolas (safras de 2001/02; 2002/03, 2003/04, 2004/05 e 2005/06).

As doses mais econômicas de calcário para a implantação do pomar de caramboleiras foram calculadas utilizando-se das informações e considerações de Raij (1991) e Zebarth et al. (1991). Neste estudo, considerou-se o preço médio do quilo da carambola in natura comercializado no CEASA - Campinas, no valor de $\mathrm{R} \$ 5,97$ referente ao ano agrícola de 2008 (CEASA, 2010). O custo da tonelada de calcário foi de $\mathrm{R} \$ 68,00$. Entretanto, acrescentaram-se os 
custos de aplicação do corretivo, que se compõe das seguintes operações: uma aração, uma gradagem e duas aplicações de calcário. Nestas operações, foram incluídos os custos da hora/máquina e do operador, perfazendo um total (calcário + aplicação) de $\mathrm{R} \$ 345,32$ (IEA, 2010). Com o objetivo de atenuar os problemas de variação cambial e de oscilação nos preços do mercado, trabalhou-se, porém, com uma relação de troca ao invés da moeda corrente, buscando-se assim dados mais estáveis. Dessa maneira, a "moeda" utilizada nos cálculos, durante todo o estudo, foi a própria carambola, considerando-se a seguinte relação de equivalência: tonelada de calcário aplicado/tonelada de carambola paga nos entrepostos, ou seja: $\mathrm{R} \$ 345,32 / \mathrm{R} \$ 5.970,00=0,05784$.

A dose mais econômica foi calculada com base na derivada da equação de regressão entre as doses de calcário aplicadas e a produção de frutos, tornando-a igual à relação de troca, ou seja: $d y / d x=$ $a_{1}+2 a_{2} x=$ relação de troca. A dose mais econômica $(x ')$ é então calculada por:

$$
x^{\prime}=\frac{a_{1}-\text { relação de troca }}{2 \cdot\left(-a_{2}\right)}
$$

Tendo em vista que o calcário foi aplicado uma única vez, em 1999, antes da implantação do pomar de caramboleiras, e que seus efeitos sobre a produção foram avaliados durante cinco anos (2002 a 2006), utilizou-se de produções acumuladas de frutos ao longo desse período para calcular as doses mais econômicas do corretivo.

\section{RESULTADOS E DISCUSSÃO}

A primeira colheita de frutos ocorreu em 2002, não apresentando diferenças significativas de produtividade entre as doses de calcário (Figura 1). Isso ocorreu, provavelmente, devido à alta variabilidade na capacidade de produção de frutíferas jovens, além do tempo necessário para que essas plantas perenes manifestem efeitos de tratamentos. Assim, não foi possível calcular a dose mais econômica de corretivo para a safra de 2002 .

A partir de 2002, as produções foram acumuladas sucessivamente, apresentando comportamento quadrático com a elevação das doses de calcário. Os resultados condizem com a lei dos incrementos decrescentes, segundo a qual, biologicamente, a produção da planta tende a se reduzir a cada aumento unitário de insumo aplicado (PIMENTEL-GOMES, 1990), logo após o ponto de inflexão da curva de resposta.

O modelo de segundo grau requer que o coeficiente $a_{1}$ seja positivo e o coeficiente $a_{2}$ seja negativo, a fim de permitir o cálculo da dose mais econômica. A máxima eficiência física do insumo pode ser obtida derivando-se a equação de regressão entre a produção de frutos e as doses de calcário, igualando-a a zero. Nesse caso, tem-se a dose que promove a máxima produção (ou ponto de máxima da equação). Porém, nenhuma consideração econômica, como o custo do corretivo ou o preço de venda do produto, é levada em conta nesse cálculo, restringindo a utilidade dessa informação para a prática agrícola.

É importante destacar que, ano após ano, os valores de produção incrementaram, tanto em função do acúmulo da produção anual, como também devido ao crescimento e desenvolvimento das caramboleiras, que aumentaram em diâmetro do tronco, altura, área foliar e volume da copa e, consequentemente, em sua capacidade de produzir frutos. Assim, as produções no ano de 2002 foram menores, independentemente da dose de calcário, comparativamente aos anos posteriores.

A Figura 1 mostra, ainda, que as caramboleiras responderam à aplicação das diferentes doses de calcário durante todo o período experimental. Desse modo, em que pese o fato de essa Oxalidaceae ser considerada uma planta pouco exigente à fertilidade do solo (DONADIO et al., 2001) e cultivada comercialmente em condições de solo ácido em seu centro de origem (Malásia) com $\mathrm{pH}=4,5$ (GREEN, 1987), a caramboleira responde com aumento de produção aos tratos culturais, como a calagem.

A caramboleira é cultivada em nossas condições com baixa tecnologia (SEKIYA; CUNHA, 1999), apresentando alta variação de produtividade (15 a 60 t ha $^{-1}$ ), segundo Bastos (2004). Desse modo, é importante destacar que, mesmo sendo um pomar jovem, as produtividades médias obtidas em todas as safras deste estudo estiveram no intervalo apresentado por Bastos (2004). Isto se deve, especialmente, ao emprego de cultivar mais produtiva, que exige, porém, manejo da calagem, da adubação e do uso de irrigação mais adequado. Assim, em empreendimentos agrícolas, a aplicação de calcário deve ser obrigatoriamente considerada, quando se pretende obter resultados satisfatórios de produtividade.

$\mathrm{O}$ valor obtido com a produção de uma cultura pode ser obtido multiplicando-se a curva de produção pelo preço de venda do produto. Do mesmo modo, uma linha de custo do corretivo pode ser gerada, multiplicando-se as doses empregadas pelo custo de cada dose correspondente. O lucro máximo ocorrerá no ponto em que se observa a máxima diferença (distância) entre a linha de custo 
do insumo e a curva de resposta da produção.

Utilizando-se da equação de regressão da Figura 1 (produção acumulada de frutos dos anos de 2002+2003), e com base nas informações de Raij (1991) e Zebarth et al. (1991), pode-se calcular a dose economicamente viável de calcário para o pomar de caramboleiras:

$$
x^{\prime}=\frac{3,6788-0,05784}{2 \cdot(0,4011)}=4,5 t h a^{-1}
$$

Assim, a dose econômica calculada para o período de $2002+2003$ foi de $4,5 \mathrm{t} \mathrm{ha}^{-1}$. A receita prevista, devido à calagem, pode ser determinada pelo aumento de produção de carambolas $=8,4 \mathrm{t}$ ha $^{-1}$ (produção de frutos com a dose mais econômica igual a $37,371 \mathrm{tha}^{-1}$, menos a produção na dose zero de calcário igual a $28,938 \mathrm{t} \mathrm{ha}^{-1}$ ), subtraindo-se o custo do calcário em toneladas de fruto $(0,3)$.

Empregando-se a mesma sistemática, podem-se calcular as doses mais econômicas para o pomar nos anos seguintes, conforme apresentado na Tabela 2, sempre considerando as produções acumuladas sucessivamente, e que o calcário foi aplicado uma única vez, apenas na instalação do experimento, em julho/agosto de 1999.

Observa-se que, para os anos de 2002/2003, o lucro, devido à aplicação do calcário, foi relativamente pequeno, comparativamente ao acumulado das demais safras. Isso é compreensível, tendo em vista que as plantas eram jovens, em início de formação e com capacidade produtiva reduzida. A partir daí, com o desenvolvimento das caramboleiras, as produções cresceram, e o lucro, devido à correção da acidez, foi evidenciado.

É importante destacar, também, que as produções acumuladas obtidas, empregando-se as diferentes doses economicamente viáveis, coincidiram com as produções máximas possíveis (Tabela 2). Isso ocorreu devido à alta capacidade produtiva desta frutífera e ao elevado preço médio da carambola praticado no mercado. O valor pago pela fruta é sazonal, sendo regulado pela oferta/ procura. De acordo com os dados da CEASA (2009), o quilo da carambola esteve entre $\mathrm{R} \$ 2,50$ e $\mathrm{R} \$ 11,50$. Mesmo considerando o valor mais baixo ( $\mathrm{R} \$ 2,50 /$ $\mathrm{kg}$ de carambola), o lucro na safra de $2002+2003$, em que a planta ainda não possuía plena capacidade produtiva, foi de $7,8 \mathrm{tha}^{-1}$ de fruto. A porcentagem da produção de frutos obtida com a dose mais econômica, em relação à produção máxima, seria de $100 \%$. Desse modo, a aplicação da dose econômica permitiu economia de calcário, sem perda significativa da produção de frutos.

Outro aspecto que deve ser considerado é que, havendo variação no tipo de solo, planta ou PRNT do corretivo empregado, haverá alteração nas doses, requerendo, pois, ajustes.

TABELA 1 - Atributos químicos do Latossolo Vermelho antes do plantio das caramboleiras, em diferentes profundidades. Bebedouro-SP, maio/1999

\begin{tabular}{|c|c|c|c|c|c|c|c|c|c|c|c|}
\hline \multicolumn{2}{|c|}{ Camada $\mathrm{pH} \mathrm{CaCl} 2$} & M.O. & $\begin{array}{c}\mathrm{P} \\
\text { (Resina) }\end{array}$ & K & $\mathrm{Ca}$ & $\mathrm{Mg}$ & $(\mathrm{H}+\mathrm{Al})$ & SB & $\mathrm{T}$ & $\mathrm{Al}$ & \multirow{2}{*}{$\frac{\mathrm{V}}{\%}$} \\
\hline $\mathrm{cm}$ & & $\mathrm{g} \mathrm{dm}^{-3}$ & $\mathrm{mg} \mathrm{dm}^{-3}$ & \multicolumn{7}{|c|}{ - } & \\
\hline $0-20$ & 4,7 & 18 & 6 & 1,3 & 9 & 4 & 40 & 14,3 & 54,3 & 8 & 26 \\
\hline $20-40$ & 4,4 & 16 & 3 & 0,8 & 6 & 4 & 41 & 10,8 & 51,8 & 11 & 21 \\
\hline $40-60$ & 4,4 & 16 & 4 & 0,6 & 7 & 4 & 45 & 11,6 & 56,6 & 12 & 20 \\
\hline $60-80$ & 4,0 & 9 & 1 & 0,3 & 7 & 3 & 58 & 10,3 & 68,3 & 11 & 15 \\
\hline
\end{tabular}

TABELA 2 - Dose econômica de calcário, calculada em função da produção acumulada de frutos da caramboleira e do custo do corretivo, para o período de 2002 a 2006.

\begin{tabular}{cccccc}
\hline $\begin{array}{c}\text { Produção } \\
\text { acumulada }\end{array}$ & $\begin{array}{c}\text { Dose } \\
\text { econômica }\end{array}$ & $\begin{array}{c}\text { Aumento de } \\
\text { produção }\end{array}$ & $\begin{array}{c}\text { Custo do } \\
\text { calcário }\end{array}$ & Lucro & Produção $^{1}$ \\
\hline & t ha $^{-1}$ & $-----------~$ & t de fruto por ha ----------- & $\%$ \\
2002 a 2003 & 4,5 & 8,4 & 0,3 & 8,1 & 100 \\
2002 a 2004 & 4,8 & 16,0 & 0,3 & 15,7 & 100 \\
2002 a 2005 & 5,3 & 28,8 & 0,3 & 28,5 & 100 \\
2002 a 2006 & 5,3 & 34,2 & 0,3 & 33,9 & 100 \\
\hline
\end{tabular}

${ }^{1}$ Porcentagem da produção de frutos obtida com a dose mais econômica, em relação à produção máxima. 


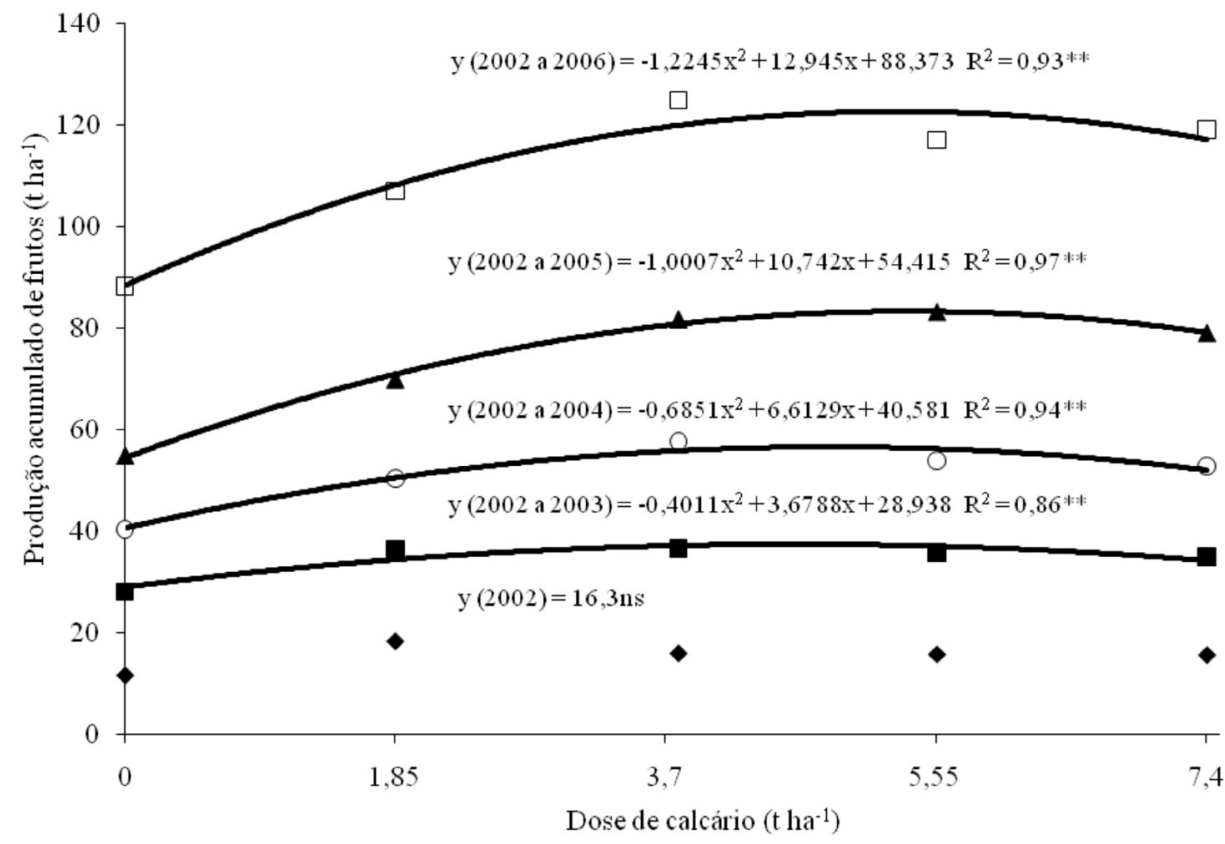

FIGURA 1- Produção acumulada de carambolas no período de 2002 a 2006, em função das doses crescentes de calcário.

\section{CONCLUSÕES}

As caramboleiras respondem positivamente à aplicação de calcário, elevando a produção de frutos durante todo o período experimental. As produções acumuladas de frutos, nos anos sucessivos de 2003, 2004,2005 e 2006, estão associadas às doses mais econômicas de calcário de 4,5; 4,8; 5,3 e 5,3 $\mathrm{t} \mathrm{ha}^{-1}$, respectivamente.

\section{REFERÊNCIAS}

BASTOS, D. C. A cultura da carambola. Revista Brasileira de Fruticultura, Jaboticabal, v.26, n. 2, 2004.

BERNARDO, S. Manual de irrigação. 6.ed. Viçosa: UFV, Imprensa Universitária, 2002. 656p.

CEASA DE CAMPINAS. Cotações. Disponível em: <http://www.ceasacampinas.com.br/cotacoes>. Acesso em: 06 out. 2010.

DONADIO, L. C.; SILVA, J. A. A.; ARAÚJO, P. S. R.; PRADO, R. M. Caramboleira (Averrhoa carambola L.). Jaboticabal: Sociedade Brasileira de Fruticultura, 2001. 81p.
EMBRAPA. Empresa Brasileira de Pesquisa Agropecuária. Centro Nacional de Pesquisa de Solo. Sistema brasileiro de classificação de solos. Brasília: Produção de Informações, 1999. 412p.

FAO - Organização das Nações Unidas para Agricultura e Alimentação. FAOSTAT Disponível em: <http://faostat.fao.org/site/567/default.aspx $>$. Acesso em: 23 mar. 2011.

GREEN, J. G. Carambola production in Malaysia and Taiwan. Proceedings Florida State Horticultural Society, Gainesville, v.100, n. 1, p.275-278, 1987.

IEA - Instituto de Economia Agrícola. In: BEMELMANS, P.F.; ROCHA, M.B. Goiaba para indústria: custos de formação e manutenção do pomar em 2002/03. Disponível em: <http://www.iea.sp.gov. br>. Acesso em: 01 out. 2010.

LEAL, R. M.; NATALE, W.; PRADO, R. M.; ZACCARO, R. P. Adubação nitrogenada na implantação e na formação de pomares de caramboleira. Pesquisa Agropecuária Brasileira, Brasília, v. 42, n. 8, p. 1111-1119, 2007.

NATALE, W.; COUTINHO, E.L.M.; BOARETTO, A.E.; PEREIRA, F.M. Goiabeira: calagem e adubação. Jaboticabal: FUNEP, 1996. 22p. 
NATALE, W.; PRADO, R. M.; ROZANE, D.E.; ROMUALDO, L.M.; SOUZA, H.A.; HERNANDES, A. Resposta da caramboleira à calagem. Revista Brasileira de Fruticultura, Jaboticabal, v. 30, n.4, p.1136-1145, 2008.

PIMENTAL-GOMES, F. Curso de estatística experimental. Piracicaba: Nobel, 1990. 468 p.

PRADO, R. M.; NATALE, W. Leaf sampling in carambola trees. Fruits, Paris, v. 59, n. 4, p. 281289, 2004 b.

PRADO, R. M.; NATALE, W. A calagem na nutrição e no desenvolvimento do sistema radicular da caramboleira. Revista de Ciências Agroveterinárias, Lages, v. 3, n. 1, p. 3-8, 2004 a.
RAIJ, B. van; CANTARELLA, H.; QUAGGIO, J. A.; FURLANI, A. N. C. (Ed.). Recomendações de adubação e calagem para o Estado de São Paulo. Campinas: Instituto Agronômico/Fundação IAC, 1997. 285p. (Boletim Técnico, 100).

SEKIYA, R. F. M.; CUNHA, R. J. P. Influência do método de extração e do armazenamento na germinação de sementes da caramboleira. Revista Brasileira de Fruticultura, Jaboticabal, v.21, n.1, p.57-59, 1999.

ZEBARTH, J.B.; SHEARD, R.W.; CURNOE, W.E. A soil test calibration method for potassium on alfafa which allows for variation in crop value and fertilizer cost. Journal of Production Agriculture, Madison, v. 4, n. 3, p. 317-322, 1991.

RAIJ, B. van. Fertilidade do solo e adubação.

Piracicaba: Ceres, Potafós, 1991. 343p. 